\title{
Ultrastructural Analysis of the Earliest Steps of Kidney Stone Formation: Insights into Novel Preventive Strategies
}

\author{
Jilei Huang, Yan Liu, Chris Petzold, Xue-Ru Wu and Fengxia (Alice) Liang
}

New York University Langone Health, New York, New York, United States

Kidney stone disease afflicts about $12 \%$ of the adult population, and the incidence and the recurrence rates are both on the rise [1]. For such a prevalent disease, prevention is the key; however, existing approaches are non-specific due largely to the fact that the earliest steps of kidney stone formation remain poorly defined [2]. We took advantage of the availability of a knockout mouse model we developed that is deficient for a major kidney-specific protein, namely Tamm-Horsfall protein (or THP) [3]. Although the knockout mice developed intra-renal calcium deposits in the papillae upon histochemistry and light microscopy, their precise location and chemical composition had not been delineated, thus hampering our ability to correlate them with the earliest lesions found in stone-bearing human kidneys. To tackle this critical issue, we carried out transmission electron microscopy (TEM) to pinpoint the ultrastructural localization of the calcium crystals and m-FTIR spectroscopy to determine the chemical components of the crystals.

The papillae of the kidneys were dissected out from twelve-month old THP-knockout mice and wild-type control mice and fixed with $2.5 \%$ glutaraldehyde and $2 \%$ paraformaldehyde prepared in $0.1 \mathrm{M}$ sodium cacodylate buffer ( $\mathrm{pH} 7.4)$ at room temperature for two hours. The fixed papillae were then cut into two halves, one half prepared for paraffin embedding and the other half processed for transmission electron microscopy (TEM). For TEM, the samples were post fixed with $2 \%$ osmium texoxide, then dehydrated with serial ethanol and propylene oxide, and embedded by either EMbed 812 or Spurr epoxy resin. For the determination of the chemical composition, kidney was fixed in $10 \%$ buffered formalin and paraffin embedded. Paraffin sections were first stained in von Kossa solution, and renal crystals were analyzed by $\mu$-FTIR spectroscope by comparing the spectra with standard spectra of calcium oxalate, calcite, and calcium phosphate.

TEM allowed us to demonstrate that most mineral deposits visible by histochemistry were actually localized to the interstitial space in the basement membrane area between the collecting ducts. Overall, Spurr preserved crystals significantly better than EMbed 812, the latter leaving many empty crystal footprints (Fig 1,2). The interstitial crystals exhibited distinct ring structures with electron dense mineral materials interspersed by electron-loose proteinaceous materials (Fig. 3). No prominent inflammatory cell infiltration was observed adjacent to the crystals. No crystals were found inside the collecting tubular epithelial cells. This needs to be further confirmed by three-dimensional electron microscopy using serial block-face scanning electron microscopy. $\mu$-FTIR showed that interstitial crystals contained mainly calcium phosphate in the form of hydroxyapatite, with a small amount of calcium carbonate. For detailed analysis the chemical composition of the smaller crystals which were beyond the detectable limit of $\mu$ FTIR, we intend to perform Energy Dispersive Spectroscopy-Scanning electron microscopy (EDS-SEM) with more sensitive and high resolution in order to provide additional insights into the earliest stage of kidney stone formation. The fact that the intra-renal crystals are interstitial, calcium phosphate in nature and lack accompanying inflammation suggests that these crystals resemble the Randall's plaques found in stone-bearing human kidneys, which could serve as a nidus for calcium oxalate to attach and accumulate to form stones. Reducing the urinary supersaturation of calcium phosphate should be further explored as an effective approach to disrupt the earliest steps and prevent the formation and/or the recurrence of kidney stones. 


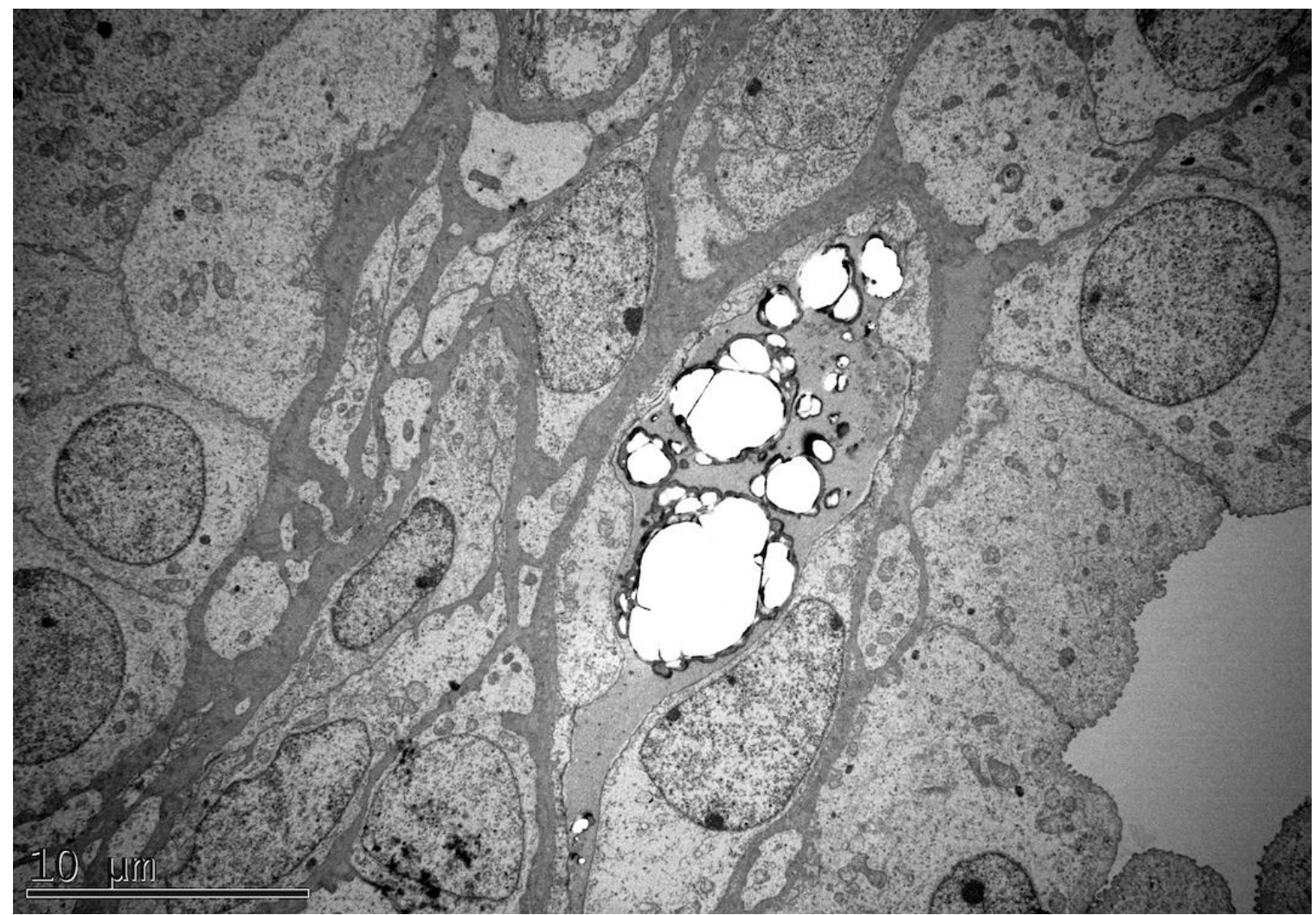

Figure 1. Intra-renal crystals were poorly preserved with the EMbed 812 embedding method, and only empty footprints were visible.
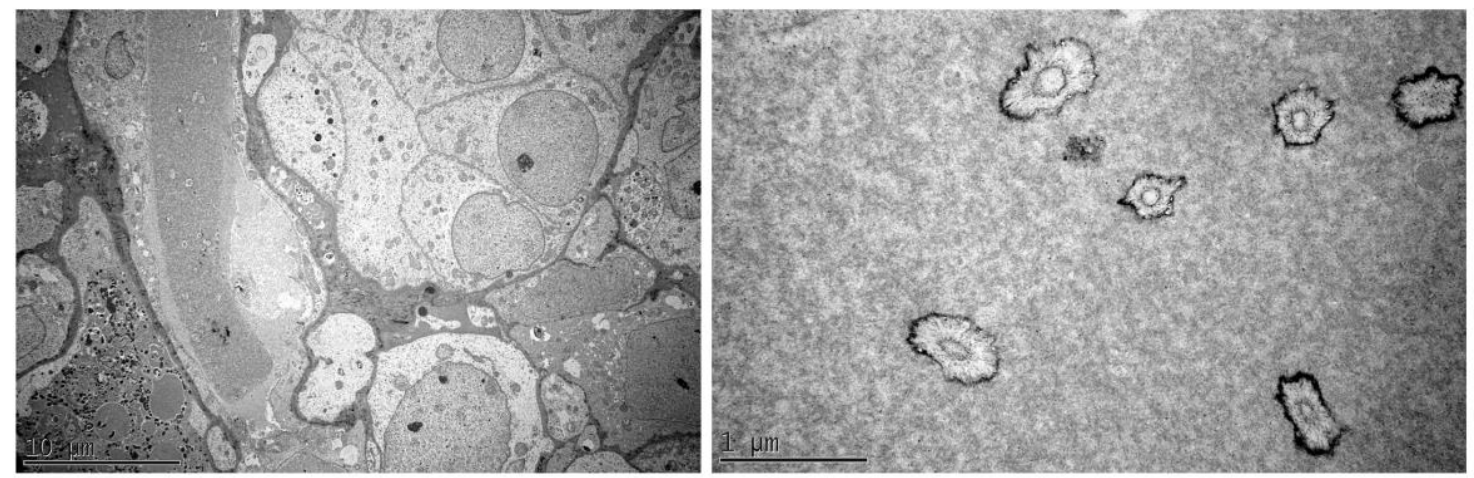

Figure 2. The crystals were well preserved in the papillae of the kidneys embedded with Spurr, and those crystals were localized in the interstitium within the renal papillae.

\section{References}

[1]T Alelign and B Petros. Adv Urol., (2018), 3068365.

[2] Saeed R. Khan et al, Nat Rev Dis Primers., (2016), 2, p.16008.

[3] L Mo et al, Am J Physiol Renal Physiol., (2007), 293(6), p.1935. 